\title{
Review of: "The ability of continuous-wave Doppler ultrasound to detect fetal growth restriction"
}

\author{
yao-lung chang ${ }^{1}$ \\ 1 Chang Gung Memorial Hospital
}

Potential competing interests: The author(s) declared that no potential competing interests exist.

1.How the resistance index (RI) be measured? Only once or multiple measurements, under what condition? Ultrasound machine? Ultrasound power, frequency? How was classified as normal or abnormal in local tertiary hospitals do not describe in the method section

2. How many cases are categorized as abnormal by low cost CWD (UmbiflowTM) and latter classified as normal in local tertiary hospitals do not describe in the method section.

3. Maternal weight and high between the normal and abnormal RI groups should be described.

4. Since the gestational age at birth ( $38.3 \pm 1.0$ vs $39.1 \pm 1.2$ ) was significantly different between normal and abnormal RI groups, the comparison of birth weight should be adjusted according to the gestational age. Because gestational age at delivery is a significant confounding factor for birth weight in Table 1. And since infant size also could be influenced by gestational age at delivery, the statistics for table 2,3 and 4 should be recalculated by adding the gestational age at delivery as a confounding factor. 